Ann. Zootech., I974, 23 (4), 423-434.

\author{
I.N.R.A. \\ BIBLIOTHEQUE UO 35906 \\ DOMaINE de CROUELLE \\ 63039 \\ CLERMONT-FD CEDEX 2
}

\title{
COMPOSITION CHIMIQUE, DIGESTIBILITÉ ET INGESTIBILITÉ D'UN MAÏS MÂLE-STÉRILE, SUR PIED ET APRES ENSILAGE
}

\author{
J. ANDRIEU et C. DEMARQUILLY \\ avec la collaboration technique de J.-M. Borssau, H. Bousquet, \\ Jacqueline Jamot, Marie Jailler et L. L'Hotelier \\ Laboratoire des Aliments, \\ Centre de Recherches de Clermont-Ferrand, I. N.R. A., \\ Theix, 63110 Beaumont
}

\section{RÉSUMÉ}

Au cours de 2 années, la digestibilité et la quantité ingérée d'un maïs mâle-stérile (variété "Sucrensilage") sur pied et après ensilage ont été mesurées sur des moutons. La plante sur pied a été étudiée à partir de la floraison et durant 9 ou ro semaines consécutives.

- En raison d'une fécondation partielle du grain, le pourcentage de grain clans la matière sèche de la plante a augmenté régulièrement mais lentement pour atteindre en fin d'étude 30 et 20 p. Ioo respectivement en 1970 et en I97I. Dans le mmêe temps, la teneur en matière sèche de la plante a augmenté lentement $(0,15$ à 0,22 point/jour) et a varié dans des limites très différentes d'une année à l'autre (de i 6 à 30 p. Ioo en I970 et de I 2 à 20 p. Ioo en I97 I). L'évolution des teneurs en matières azotées et en cellulose brute avec l'âge de la plante a été faible.

- la digestibilité de la matière organique de la plante sur pied, dont l'évolution a été lente surtout durant les 6 ou 7 premières semaines, a été en moyenne pour les deux années de $65,8 \mathrm{p}$. Ioo (soit $0,6_{4} \mathrm{UF} / \mathrm{kg}$ de matière sèche). Cette valeur est inférieure de 8 p. roo à celle enregistrée en moyenne pour les 20 maïs fertiles étudiés au laboratoirc.

- la quantité de matière sèche ingérée par le mouton a été élevée $\left(62,9 \mathrm{~g}\right.$ de $\left.\mathrm{MS} / \mathrm{kg} \mathrm{P}^{0,75}\right)$ et supérieure de 20 p. Ioo à celle mesurée pour les maïs fertiles. Cependant la quantité ingérée, dont l'évolution a été très différente d'une année à l'autre a été nettement plus faible en I97 I qu'en I970 (59,3 contre 66, I g) en raison vraisemblablement d'une teneur en matière sèche trop faible.

- la composition du mélange des acides gras volatils du jus de rumen des moutons qui ont reçu le Sucrensilage en vert a été assez différente de celle enregistrée avec les maïs fertiles : la teneur en acide acétique a été plus élevée et celle en aciđe propionique plus faible dans le cas du maïs stérile.

- les quantités de matière sèche et d'UF récoltées à l'hectare ont augmenté durant les 5 ou 6 semaines qui ont suivi la floraison pour se stabiliser vers 17 tonnes et I I ooo UF en I970 et de 19 tonnes et 13000 UF en I971. La quantité de matières azotées digestibles a atteint son maximum beaucoup plus tôt (durant les 3 premières semaines après la floraison). L'exploitation en vert du Sucrensilage ne devrait donc pas se situer avant la $3^{\mathrm{e}}$ ou $4^{\mathrm{e}}$ semaine après la floraison : 
la plante est alors plus riche en matière sèche et les productions de matière sèche et d' $\mathrm{L}^{\mathrm{F}} \mathrm{F} / \mathrm{ha}$ ont pratiquement atteint leur maximum.

- les caractéristiques fermentaires des ensilages, que nous avons réalisés 5 et 8 semaines après la floraison, ont été voisines de celles observées pour les maïs fertiles, mais les pertes durant la conservation ont été en moyenne plus importantes.

- l'ensilage n'a cntraîné aucune diminution de la digestibilité de la matière organique mais s'est traduit par une diminution d'ingestibilité plus importante que celle enregistrée dans le cas des maïs fertiles (- 16,5 contre -6 p. Ioo). L'ensilage de maïs stérile, moins riche en énergie et ingérée en quantité comparable, aura donc une valeur alimentaire inférieure à celle d'un mais fertile.

\section{IN'TRODUCTION}

La valeur alimentaire de la plante de maiss normale sur pied ou ensilée, ainsi que ses facteurs de variation, sont maintenant bien connus à la suite des travaux réalisés tant à l'étranger (revue de Coppock et STONE, I968) qu'en France (DEMarQUili.y, I969; ANDRIEU et DeMarquiLly, I974 $a$ et $b$ ). En revanche, les résultats concernant la valeur alimentaire des maïs ne formant pas de grain sont relativement peu nombreux (Bratzler et al., I965; Cummins et MCCUllough, I97I; Metske et Goodrich, ig66; Perry et Caldwell, ig69; Stake et al., I973). Or, depuis quelques années, des variétés de maïs stériles existent sur le marché français. Décrites comme étant très bien consommées en raison de leur teneur élevée en glucides solubles, ces variétés permettraient d'obtenir des productions de matière sèche et d'éléments nutritifs par hectare égales sinon supérieures à celles de maïs normaux. Dans le cadre général des études entreprises à la Station de Recherches sur l'Élevage des Ruminants sur la valeur alimentaire du maïs fourrage, nous avons étudié chez la variété mâle-stérile Sucrensilage, l'évolution de la composition, de la valeur nutritive et de l'ingestibilité (c'est-à-dire de la quantité de matière sèche volontaisement ingérée par l'animal) de la plante sur pied et de la plante ensilée.

\section{MATÉRIEL, ET MÉTHODES}

La variété de maïs mâle-stérile "Sucrensilage " a été semée le 4 mai I97o à Malintrat (altitude $35^{\circ} \mathrm{m}$ ) et le 13 mai I97 I à Chanonat (altitude $500 \mathrm{~m}$ ). En r970 et I97I les peuplements ont été respectivement de 49700 et 96400 pieds/ha. Les fertilisations apportées ont été de I $20 \mathrm{~kg}$ d'N et $90 \mathrm{~kg}$ de $\mathrm{P}$ dans le premier essai et de $\mathrm{I} 20 \mathrm{~kg}$ d' $\mathrm{N}$ et roo $\mathrm{kg}$ de $\mathrm{P}$ dans le deuxième essai. Variété demi-tardive (fin du groupe 3), le "Sucrensilage " a fleuri le 3 août I970 et le I 2 août I97 I. Dans ces deux essais, le "Sucrensilage a été partiellement fécondé soit par son propre pollen, soit par celui de maïs fertiles de précocités pourtant très différentes (groupe o et I), semés à la même date dans la même parcelle. Le "Sucrensilage " n'ayant pas fait l'objet d'une comparaison très complète avec ces maïs fertiles, les résultats le concernant seront donc parfois comparés à ceux obtenus antérieurement sur 20 maïs normaux (groupe de précocité 0,1 et 2 ) étudiés en vert au Laboratoire (DEmarquilly et ANDrieu, I973).

\section{Mesures sur la plante sur pied}

La date de la floraison femelle a été déterminée sur des plantes choisies dans un endroit représentatif de la culture (Io mètres d'un rang). Elle correspond au jour où 50 p. Ioo des épis présents sur ces ro mètres avaient au moins une soie sortie. 
Chaque année et pendant 9 ou ro semaines à partir de la floraison, nous avons tout d'abord étudié l'évolution de la composition morphologique de la plante. Pour cela, nous avons, en I970, prélevé chaque semaine deux échantillons de 5 plantes, l'un pris au hasard et l'autre ne comportant que des plantes non fécondées. En I97I, nous avons réalisé un prélèvement hebdomadaire de 10 plantes prises au hasard. Ces plantes ont été ensuite séparées en grains, rafles, pédoncules, spathes, limbes et tiges + gaines.

Pour les mesures de valeur alimentaire, le "Sucrensilage " a été récolté 3 fois par semaine, haché dans un hache-paille en brins de 2 à $3 \mathrm{~cm}$ de longueur et stocké en chambre froide $\left(+4^{\circ} \mathrm{C}\right)$ jusqu'à la distribution aux animaux. Les surfaces et les quantités fauchées ont été mesurées à chaque récolte. Le maîs a été distribué à volonté ( 5 à Io p. Ioo de refus), à raison de 3 repas/jour $\left(8 \mathrm{~h} 30, \mathrm{I}_{3} \mathrm{~h} 30\right.$ et $\left.\mathrm{I} 7 \mathrm{~h} 30\right)$, à un lot de 6 moutons en cage à métabolisme. La digestibilité et l'ingestibilité ont été mesurées en continu, chaque période de mesure allant du lundi au samedi. En outre, en I97I, un mouton supplémentaire muni d'une fistule du rumen nous a servi à prélever du jus de rumen une période de mesure sur deux.

\section{Mesures sur la plante ensilée}

A partir des maïs étudiés en vert, nous avons préparé chaque année 3 ensilages. En I970, la plante a été fauchée à deux dates, le 9 septembre et le 2 octobre, soit respectivement 5 et 8 semaines après la floraison. En I971, la plante a été fauchée le 20 septembre et le 4 octobre, soit respectivement 6 et 8 semaines après la floraison. Le fourrage a été récolté avec une récolteuse chargeuse à tambour hacheur (type New-Holland $7 \mathrm{I} 7$ ) réglée pour assurer un hachage en brins de 0,5 à $\mathrm{I} \mathrm{cm}$, et a été ensilé immédiatement dans des petits silos tours de $+\mathrm{m}^{3}$ en butyl. Il a été tassé au pied au fur et à mesure du remplissage, à la suite de quoi les enveloppes de butyl ont été simplement refermées. Chaque année, la plante récoltée au $2^{\mathrm{e}}$ stade a été ensilée seule ou avec addition de ro $\mathrm{kg} /$ tonne de produit frais, d'un mélange solide contenant $50 \mathrm{p}$. Ioo d'urée et $50 \mathrm{p}$. Ioo de minéraux (phosphate bicalcique, carbonate de calcium et oligoéléments). Les quantités de matière sèche mises dans les silos et retirées des silos ont été mesurées. Durant l'hiver qui a suivi leur récolte, les ensilages ont été distribués seuls et à volonté à un lot de 6 moutons afin de mesurer lcur digestibilité et leur ingestibilité.

\section{Analyses}

Les échantillons représentatifs des fourrages verts ou des ensilages offerts aux moutons, des refus et des fèces correspondant à chaque période de mesure ont été analysés pour déterminer leurs teneurs en cendres, en matières azotées et en cellulose brute Weende.

Ces mêmes analyses, ainsi que celles concernant les teneurs en glucides solubles et en amidon, ont été également réalisées sur des échantillons prélevés à la mise en silo et représentatifs du fourrage ensilé.

Au début et à la fin de chaque période de mesure sur les ensilages, nous avons prélevé un échantillon représentatif de l'ensilage destiné aux moutons. Nous avons déterminé sur ces échantillons frais la teneur en azote et, sur leur jus, le pH, les teneurs en ammoniac (CoNwAY, I957), en acide lactique (BARKER et SUMMERSON, I94I) et en acides gras volatils (RIGAUD et JOURNET, I970).

Nous avons prélevé, 3 heures après la distribution du premier repas de la journée et durant 2 jours consécutifs, du jus de rumen sur le mouton muni d'une fistule du rumen. Le pH a été déterminé immédiatement et les échantillons ont été conservés (après addition de ro p. Ioo d'acide formique) à - I $5^{\circ} \mathrm{C}$ jusqu'au moment de la détermination en chromatographie en phase gazeuse (RIGAUD et JoURNET, I970) de leur teneur en acides gras volatils.

\section{RÉSULTATS}

\section{La plante sur pied}

En raison de la fécondation partielle des épis, la part relative des divers organes dans la matière sèche de la plante a évolué lentement, mais cependant régulièrement, durant les 9 ou ro semaines d'étude. C'est ainsi que la proportion d'épis (avec les 
spathes) dans la plante est passée de ro p. Ioo à la floraison à environ $5^{\circ} \mathrm{p}$. Ioo en I970 et 40 p. IOO en I97I, 9 ou Io semaines plus tard (fig. I). A ce dernier stade, le grain représentait respectivement 30 et $20 \mathrm{p}$. Ioo de la matière sèche.
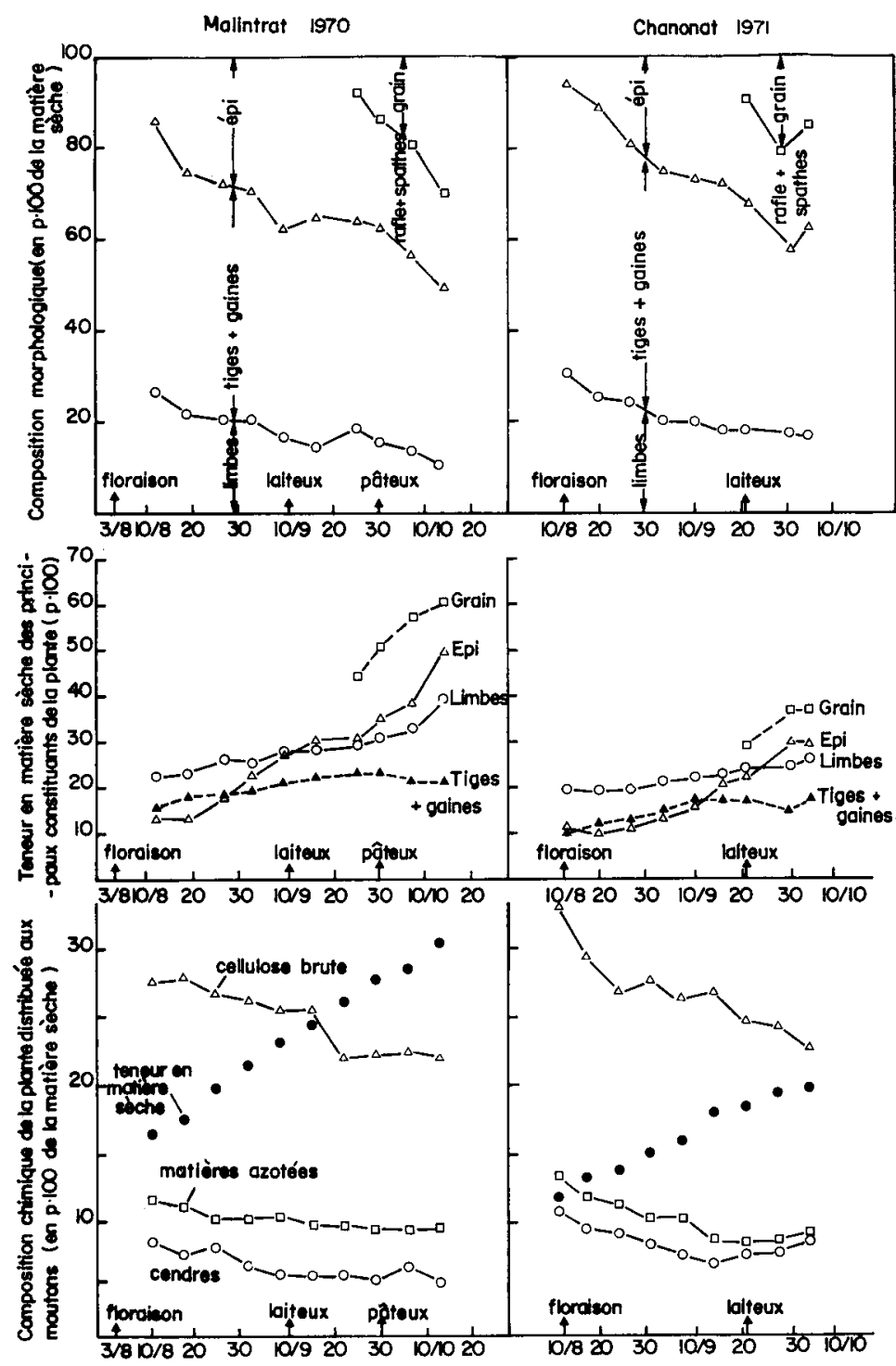

FIG. I. - Kivolution de la composition morphologique et chimique de la plante en fonction de son stade de végétation

La teneur en matière sèche de la plante a augmenté plus lentement que dans le cas des maïs précoces et fertiles semés à proximité (de $0, I_{5}$ à 0,22 point/jour contre 0,35 point) ; elle est passée de I6,6 à 30,6 p. Ioo en I970 et de II,9 à I9,7 p. Ioo en I97I. Les teneurs en cendres et en matières azotées ont diminué très lentement 
avec l'âge de la plante (fig. I). Il en a été de même en ce qui concerne la teneur en cellulose brute, sauf en I97 I où la diminution a été rapide durant les deux premières semaines d'étude. A une teneur en matière sèche du grain comparable et voisine de 30-35 p. IoO (consistance laiteuse), nous n'avons observé en I97I aucune différence de teneurs en cendres et en matières azotées entre la plante entière de "Sucrensilage " et celle d'un mais précoce cultivé dans le même champ. Par contre, la teneur en cellulose brute de la variété "Sucrensilage " a été supérieure d'environ I 8 p. IOO à celle de ce même maïs.

\section{Digestibilité.}

La digestibilité de la matière organique a augmenté très lentement durant les Io semaines d'étude : elle est passée de 64-65 p. Ioo à 68-69 p. Ioo (fig. 2). Cette dernière valeur correspond à la limite inférieure des digestibilités que nous avons mesurées sur 20 maïs fertiles étudiés au laboratoire des Aliments.

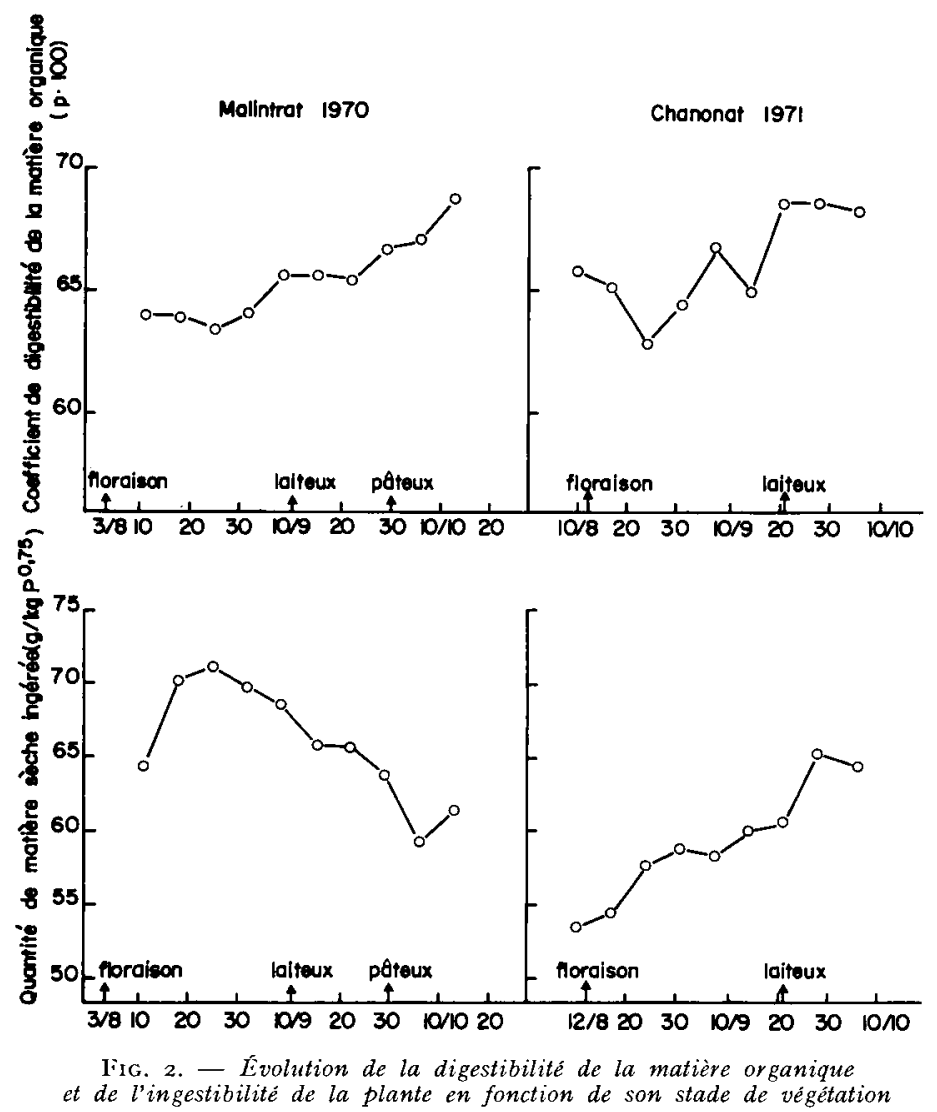

En I97r, elle a été plus variable d'une semaine à l'autre en raison vraisemblablement d'une plus grande hétérogénéité dans le taux de fécondation des épis.

La digestibilité de la cellulose brute a été systématiquement plus élevée en 


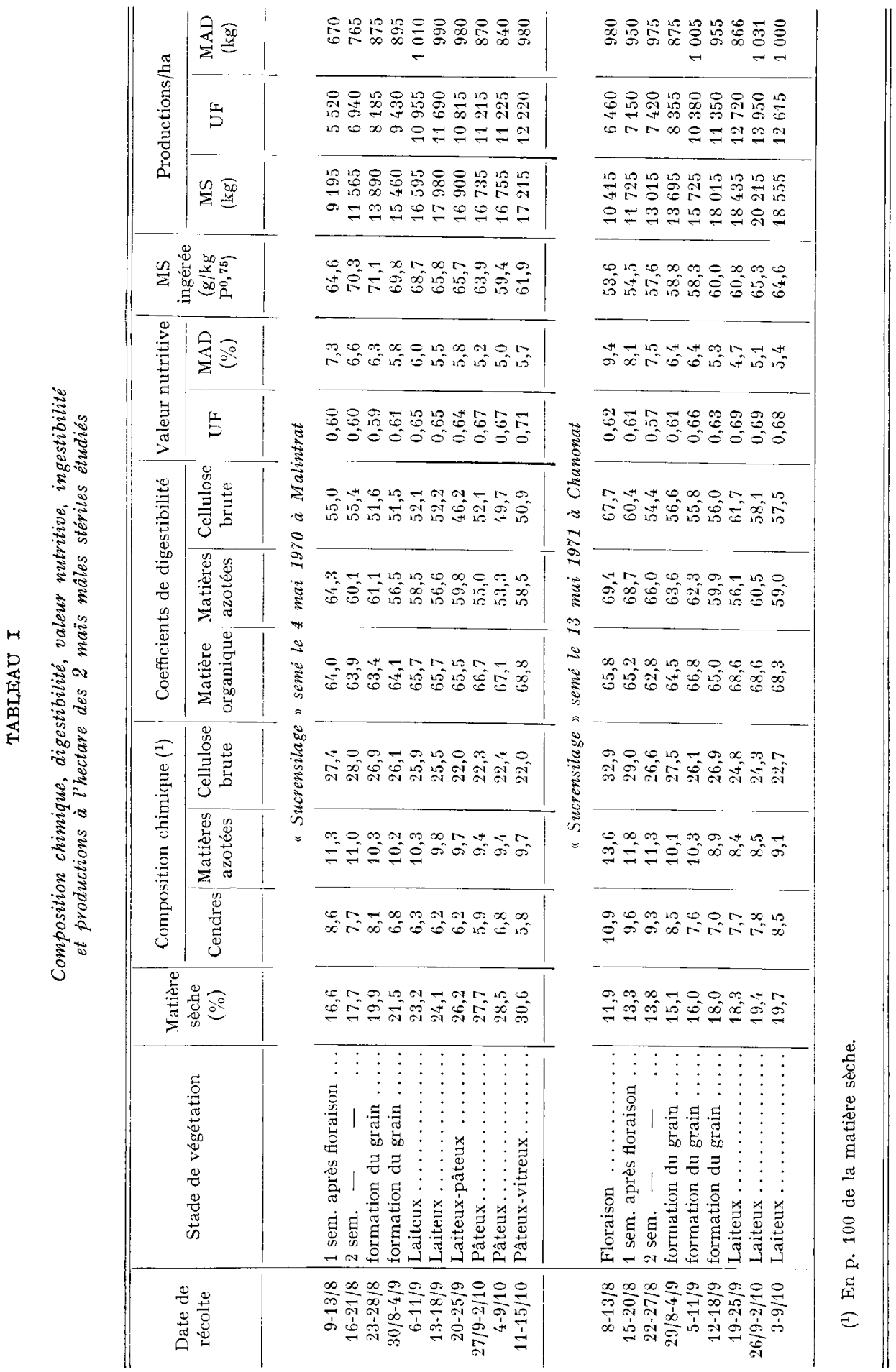


I97I qu'en I970 (en moyenne 58,7 contre $5 \mathrm{I}, 7 \mathrm{p}$. IOO) et a diminué très lentement avec l'âge de la plante (tabl. I).

La digestibilité des matières azotées a été également plus élevée en I97I (en moyenne 62,8 contre $58,4 \mathrm{p}$. Ioo) ; elle a varié parallèlement à la teneur en matières azotées de la ration. Les teneurs en matières azotées non digestibles ont été en I970 et en I97 I respectivement de 4,2 $\pm 0,2$ p. Ioo et $3,7 \pm 0,2$ p. roo.

\section{Ingestibilité et valeur alimentaire.}

L’ingestibilité de la variété "Sucrensilage " est élevée et supérieure en moyenne à celle que nous avons mesurée sur les 20 maïs précoces étudiés au Laboratoire des Aliments $\left(62,9\right.$ contre $52,5 \mathrm{~g}$ de $\left.\mathrm{MS} / \mathrm{kg} \mathrm{P}^{0,75}\right)$. Son évolution avec l'âge de la plante a été très différente suivant les années (fig. 2). En I970, elle a diminué de 70 à $60 \mathrm{~g}$ vraisemblablement parce que la plante de "Sucrensilage " qui était bien consommée au début en raison de sa teneur en matière sèche relativement élevée et de sa richesse en glucides solubles, est devenue progressivement peu différente d'un maïs normal. En I97I, elle a été en moyenne nettement plus faible qu'en I970 (59,3 contre 66,I g) et a varié d'une manière comparable à celle d'une repousse de graminée fourragère : elle a augmenté avec la teneur en matière sèche alors que la composition et la valeur nutritive de la plante ne variaient pratiquement pas.

La valeur alimentaire (c'est-à-dire la quantité de matière organique digestible ingérée) de la variété "Sucrensilage " a été élevée et pratiquement constante en I970 (en moyenne $40,3 \pm \mathrm{I}, 7 \mathrm{~g} / \mathrm{kg} \mathrm{P}^{0,75}$ ); en I97I, par contre, elle est passée de 3I,4 à $40,4 \mathrm{~g}$ durant les ro semaines d'étude. A titre de comparaison, elle a été de $35,3 \pm 4,7 \mathrm{~g}$ dans le cas des 20 maïs sur pied étudiés au Laboratoire.

\section{Composition du mélange des acides gras volatils du jus de rumen.}

Le $\mathrm{pH}$ et la concentration en acides gras volatils du jus de rumen du mouton recevant le "Sucrensilage " cultivé en I97 I ont été en moyenne respectivement de $6,3 \pm 0, \mathrm{I}$ et de 74,0 $\pm 7,9$ mMoles/ 1 de jus. La concentration du jus de rumen a augmenté parallèlement à la teneur en matière sèche et à la quantité de matière organique digestible ingérée par le mouton. Les proportions molaires d'acides acétique, propionique, butyrique et valérianique ont été respectivement en moyenne de 63,$8 ; 24,2$; II,9 et $0,1 \mathrm{p}$. Ioo. Elles ont peu varié suivant le stade de végétation de la plante distribuée à l'animal sauf durant les quinze jours qui ont suivi la floraison : la proportion d'acide acétique a diminué alors que 78,6 à 63,5 p. Ioo au profit essentiellement de la teneur en acide propionique qui a augmenté de I3, I à 23,2 p. IoO. Même si on excepte les valeurs obtenues durant cette période là, la composition du mélange des acides gras volatils semble légèrement différente de celle trouvée dans le jus de rumen des animaux recevant un maïs normal : avec une ration de "Sucrensilage ", le pourcentage molaire moyen d'acide acétique est supérieur (60,I contre 55,2 p. roo) alors que celui d'acide propionique est inférieur $(26,9$ contre 29,6 p. IO0).

\section{Productions à l'hectare.}

Les quantités de matière sèche et d'UF récoltées à l'hectare ont augmenté rapidement et régulièrement après la floraison pour être maximum environ 5 ou 6 semaines plus tard (fig. 3) ; le grain était alors au stade laiteux. Cependant, lesi 
plantes non fécondées ont atteint leur poids sec maximum plus tôt (environ 3 semaines après la floraison). Les quantités maximum produites/ha ont été en I970 de I 7 tonnes et de II 000 UF et en I97I de I9 tonnes et 13000 UF. A titre indicatif, les variétés $L G 11$, Funk's G 245 et INRA 258 semées en 1970 dans le même champ à des peu-
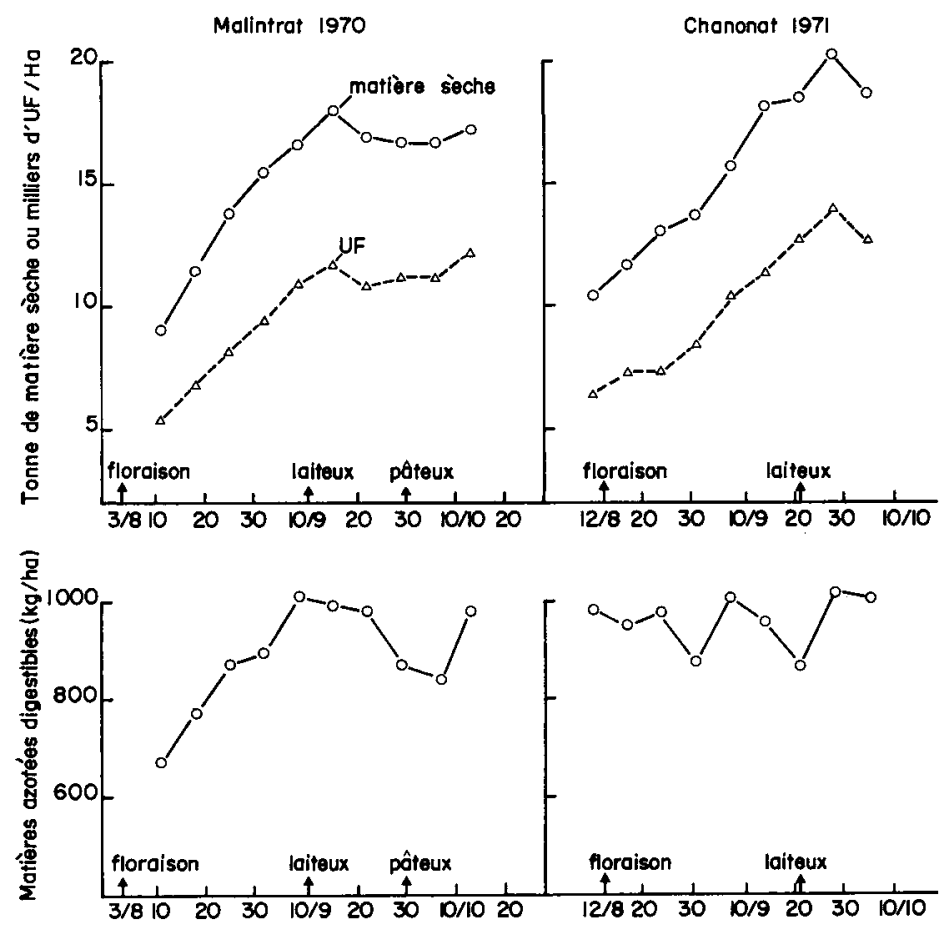

FIG. 3. - Evolution de la production de matière sèche, d'UF et de matières azotées digestibles à l'hectave en fonction du stade de récolte

plements compris entre 70000 et 98 ooo pieds/hectare ont produit au stade vitreux respectivement $18,3, \mathbf{r} 6,0$ et 14,6 tonnes de matière sèche/ha et 13250 , 12050 et Io 950 UF/ha. De même, en I97I la variété Junior 160 semée dans le même champ à un peuplement de 90000 pieds/ha a produit 17,0 tonnes de matière sèche et 12150 UF/ha. Quant aux quantités de matières azotées digestibles récoltées à l'hectare, elles ont atteint leur maximum environ 2 à 3 semaines après la floraison dans le premier essai et dès la floraison dans le $2^{\mathrm{e}}$ essai (fig. 3). Flles ont été alors importantes (de l'ordre de 900 à $\mathrm{I} 000 \mathrm{~kg}$ ) et supérieures de 4 à $\mathrm{Io} \mathrm{p}$. Ioo à celles produites par les maïs normaux cultivés dans le même champ.

\section{La plante ensilée}

Malgré une teneur en matière sèche faible (tout au moins lorsque le grain se développe peu ou pas du tout), la plante de "Sucrensilage " s'ensile bien parce qu'elle est très riche en glucides solubles (I 7 à $22 \mathrm{p}$. Ioo de la matière sèche, tabl. 2). Ces glucides solubles, qui n'ont pas été entièrement fermentés (il en restait en moyenne 


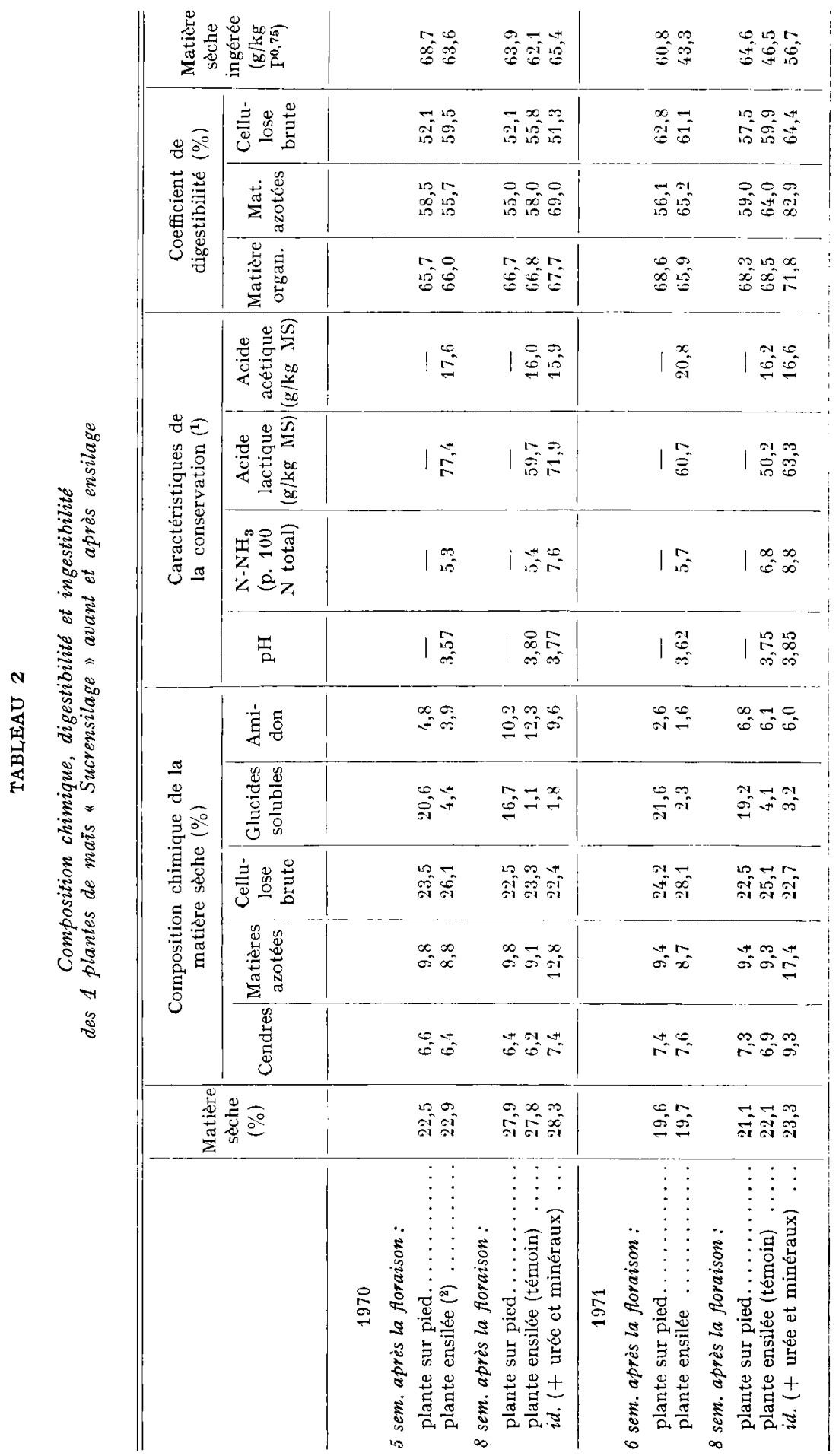


2,8 p. roo dans la matière sèche des ensilages), ont donné naissance essentiellement à de l'acide lactique (en moyenne $64 \mathrm{~g} / \mathrm{kg}$ de MS) et à un peu d'acide acétique (en moyenne $17 \mathrm{~g} / \mathrm{kg}$ de MS). Les 7 ensilages ont eu un $\mathrm{pH}$ particulièrement faible $(3,6$ à 3,8$)$ et ont donné lieu à une ammoniogenèse très réduite $(5,3$ à $8,8 \mathrm{p}$. Ioo de l'azote étaient sous forme d'ammoniac). Si la nature des fermentations est donc très comparable à celle décrite dans le cas des ensilages de plantes entières de maïs normaux, leur intensité correspond mieux à celle observée dans le cas des ensilages de cannes de maïs (ANDRIEU et DEMARQUILLY, I974 a).

Par contre, les pertes de matière sèche totales corrigées (jus, gaz, inconsommable) ont été en moyenne plus élevées (I3,2 contre 9,I p. IOo) que celles enregistrées dans le même type de silo avec des ensilages de maïs normaux.

\section{Digestibilité et ingestibilité.}

Pour les 4 ensilages réalisés sans urée, la diminution de digestibilité entre la plante sur pied et la plante ensilée a été, comme dans le cas de maïs normaux ( $\mathrm{AN}$ DRIEU et DEMARQUILLY, I974 $a$ ), très faible, voire nulle : en moyenne - 0,5 ou - I,8 points selon qu'on tient compte ou non des pertes de produits volatils lors du séchage à l'étuve (tabl. 2).

L,es différences de digestibilité des matières azotées entre la plante sur pied et la plante ensilée ont été liées étroitement à des différences de teneurs en matières azotées de la ration. A une exception près, la digestibilité de la cellulose brute des ensilages a été comparable à celle de la plante sur pied correspondante.

L'ingestibilité des 4 ensilages sans additif, qui a été en moyenne de 54,0 g de

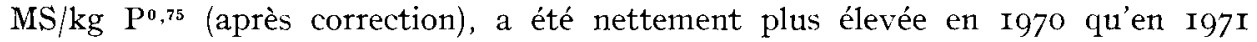
$(62,8$ contre $44,9 \mathrm{~g})$. La diminution d'ingestibilité entraînée par la conservation a été en moyenne de ro,6 g (soit I6,5 p. IOO) et variable d'un essai à l'autre $(-5,2$ p. Ioo en 1970 contre - 28,4 p. Ioo en 1971). La diminution plus importante observée en I97I s'explique par une teneur en matière sèche plus faible et par le fait que la teneur en azote de l'ensilage sans additif était un facteur limitant de son ingestibilité : en effet, l'ingestibilité de l'ensilage correspondant avec urée lui a été supérieure de 22 p. Ioo (tabl. 2).

\section{DISCUSSION ETT CONCLUSION}

Par rapport à un maïs fertile ayant poussé dans des conditions normales et exploité entre le stade " début formation du grain " et la maturité, le maiss mâlestérile se caractérise par une valeur nutritive plus faible : la digestibilité de la matière organique et la valeur énergétique ont été en moyenne de $65,8 \mathrm{p}$. 1oo et $0,64 \mathrm{UF} / \mathrm{kg}$ de matière sèche dans le cas de deux maïs mâles-stériles et de 71,5 p. Ioo et 0,77 UF dans le cas des 20 maïs normaux que nous avons étudiés en vert jusqu'ici (DEmarQUILLY et ANDRIEU, I973). L,es travaux étrangers portant sur la comparaison entre les maïs mâles-stériles et les maïs normaux sont peu nombreux et ne concernent, du moins à notre connaissance, que la plante ensilée. Leurs conclusions sont souvent contradictoires en raison d'un taux de fécondation du maïs mâle-stérile très variable (Bratzler et al., I965; Cummins et McCuli.ough, I97I; Meiske et Goodrich, I966; Perry et CALDWeli, I969; STAKe et al., I973). Cependant, les études dans 
lesquelles cette comparaison a été faite dans des conditions rigoureuses (isolement de la culture afin d'éviter toute fécondation), concluent généralement à la supériorité du maïs fertile, mais cette supériorité est parfois non significative (Cummins et McCullough, I97I) ou, si elle est significative, reste faible $(+5,5$ p. Ioo ; BratzLER et al., I965).

Par contre, l'ingestibilité du maìs mâle-stérile sur pied est élevée et supérieure (en moyenne de $20 \mathrm{p}$. IOo) à celle des 20 maïs fertiles que nous avons étudiés. En fait, cette supériorité, observée globalement, pourra être d'importance très variable suivant tout d'abord les conditions de milieu (lieu-année) qui conditionnent pour une large part l'ingestibilité de la plante de maïs sur pied (DEMARqUILLY et ANDRIEU, I973). Ensuite, cette supériorité pourra être d'autant plus réduite, voire nulle, que la teneur en matière sèche du maïs mâle-stérile sera plus faible. En effet, toutes choses étant égales par ailleurs, tout se passe comme si l'eau contenue dans la plante avait un pouvoir encombrant et limitait de ce fait la quantité ingérée, du moins quand la teneur en eau du fourrage est supérieure à 82 p. IOO (VÉRITÉ et JoURNET, I970).

La plante de maîs mâle-stérile est intéressante pour l'affouragement en vert car sa valeur nutritive varie peu après la floraison et son ingestibilité est élevée, tout au moins lorsque sa teneur en matière sèche est supérieure à $\mathrm{r} 8 \mathrm{p}$. Ioo environ. Son exploitation en vert ne devrait pas se situer avant la $3^{\mathrm{e}}$ ou la $4^{\mathrm{e}}$ semaine suivant la floraison : plus riche en matière sèche, surtout si elle a été partiellement fécondée, la plante sera alors très bien consommée et les productions maximum de matière sèche et d'UF seront atteintes.

En revanche, son utilisation sous forme d'ensilage semble moins intéressante. Les caractéristiques fermentaires sont bonnes mais les pertes de matière sèche en cours de conservation sont plus importantes que pour un maïs normal par suite d'une teneur en matière sèche plus faible. L'ensilage de maïs stérile étant moins riche en énergie $(0,67$ contre $0,77 \mathrm{UF} / \mathrm{kg}$ de MS) et ayant une ingestibilité comparable, sa valeur alimentaire sous forme d'ensilage sera inférieure à celle d'un maîs fertile ayant poussé dans de bonnes conditions de végétation.

En définitive, un certain taux de fécondation étant souhaitable pour augmenter la teneur en matière sèche de la plante, sa valeur énergétique et la production de matière sèche à l'hectare, on peut se demander dans quelle mesure un maìs normal semé à un peuplement élevé, n'aurait pas donné des résultats comparables.

Rę̧u pour publication en juin 1974.

\section{SUMMARY}

CHEMICAL, COMPOSITION, DIGESTIBILITY AND VOLUN'TARY INTAKE OF FRESH AND ENSILED MALE STERILE MAIZE

During the course of two years, the digestibility and voluntary intake of fresh and ensiled male sterile maize (variety "Sucrensilagen) were determined in sheep. The fresh plant was studied from flowering for 9 or ro consecutive weeks.

The grain percentage of the plant dry matter increased regularly but slowly because of a partial fecundation of the grain, and reached, at the end of the study, 30 and 20 p. 100 in' $197^{\circ}$ and $197 \mathrm{I}$, respectively. At the same time, the dry matter content of the plant slowly increased 
(o. I5 to 0.22 percentage points/day) and varied within very different limits from one year to another (from 16 to 30 p. Ioo in 1970 and from 12 to $20 \mathrm{p}$. 100 in $197 \mathrm{I}$ ). The crude protein and crude fiber contents increased only slightly with the age of the plant (fig. I).

The digestibility of the organic matter of the fresh plant increased only slowly especially during the first 6 or 7 weeks (fig. 2, table I), and showed a mean value of 65.8 p. Ioo for the two years (i.e. $0.64 \mathrm{FU} / \mathrm{kg}$ dry matter). This value was $8 \mathrm{p}$. roo lower than that recorded, on an average, for the zo fertile maize plants studied in the laboratory.

The dry matter intake of the sheep was high $\left(62.9 \mathrm{~g} \mathrm{IM} / \mathrm{kg} \mathrm{W}^{\mathbf{0} .75}\right)$ and exceeded by $20 \mathrm{p}$. I 00 that measured in the case of fertile maize plants. However the amount ingested, the variation of which was very different from one year to another (fig. 2, table I), was markedly lower in I97I than in $197^{\circ}(59.3$ versus 66. I g) probably because the dry matter content was too low.

The composition of the volatile fatty acid mixture of the rumen fluid in sheep fed the fresh "Sucrensilage " was rather different from that recorded with fertile maize, $i . e$. higher acetic acid content and lower propionic acid content in the case of sterile maize.

The amounts of dry matter and feed units (FU) harvested per hectare increased during the 5 or 6 weeks following flowering and stabilized around I 7 tons and I I ooo $I^{i} U$ in I970 and I9 tons and 13 ooo FU in I97I. The maximum amount of digestible crude protein was reached much earlier (during the first 3 weeks after flowering). Thus zero grazing of "Sucrensilage " should not take place before the 3 rd or ${ }_{4}$ th week after flowering, at the time when the plant is more rich in dry matter and when the production of dry matter and of FU/ha have almost reached their maximum values.

The fermentation characteristics of the silages made 5 and 6 weeks after flowering, were similar to those from fertile maize (table 2 ), but losses during storage were, on average, larger.

The ensiling did not cause any decrease in the digestibility of the organic matter, but led to a greater lowering of the voluntary intake than that recorded in the case of fertile maize (I6.5 versus 6. $\mathrm{p}$ Ioo). The net effect is that practically the same quantity of sterile maize silage is ingested as of fertile maize silage, but, being lower in energy, its feed value (measured by the $F \mathbf{T}$ or DOM intake) is lower.

\section{RÉFÉRENCES BIBLIOGRAPHIQUES}

Andrieu J., Demarquilly C., I974a. Valeur alimentaire du mais fourrage. II. Influence du stade de végétation, de la variété, du peuplement, de l'enrichissement en épis et de l'addition d'urée sur la digestibilité et l'ingestibilité de l'ensilage de mais. Ann. Zootech., 23, I-25.

Andrieu J., Demaroullly $C_{\text {., I }} 974$ b. Valeur alimentaire du maïs fourrage. III. Influence de la composition et des caractéristiques fermentaires sur la digestibilité et l'ingrestibilité des ensilages de maîs. Ann. Zootech., 23, 27-43.

Barker S. B., Summerson W. H., r94r. The calorimetric determination of lactic acid in biological material. J. Biol. chem., 137, 535 .

Bratzler J.W., King T.B., Гhomas W. I., I965. Nutritive value of high-sugar corn silage. $J$. Anim. Sci, 24, I218 (Abstr.).

Conway E. J., I957. Microdiffusion analysis and volumetric error. London : Crosby, Lockwood, London.

Coppock C. E., Stone J. B., I968. Corn silage in the ration of dairy cattle : a review. Cornell Miscellaneous, Bulletin 89 .

Cummins D. G., McCullough M. E., i971. Comparison of male sterile and male fertile corn for silage. A gron. J., 63, 46-47.

Demarquilly C., I969. Valeur alimentaire du maïs fourrage. I. Composition chimique et digestibilité du mais sur pied. Ann. Zootech., 18, 17-32.

Demarquilly C., Andriev J., r973. Valeur nutritive et utilisation par les bovins de la plante entière de mais verte, ensilée ou déshydratée. $24^{\circ}$ réunion de la F.E. $Z$.

Meiske J. C., Goodrich R. D., 1966. Regular corn vs. High-sugar corn vs. Sorgo-Sudan silage. J. Anim. Sci., 25, I270 (Abstr.).

Perry T. W., Caldwell D. M., 1969. Comparative nutritive value of silages made from high-sugar male sterile hybrid corn and regular starchy corn. J. Dairy Sci., 52, IIIg-II2x.

Rigaud J., Journet M., I97o. Méthode de dosage des acides gras volatils dans le liquide du rumen. Ann. Biol. anim. Bioch. Biophys., 10, I5I-I57.

Stake P. E., Owens M. J., Schingoethe D. J., Voelker H. H., I973. Comparative feeding value of high-sugar male sterile and regular dent corn silages. J. Dairy Sci., 56, I439-1444.

Verité R., Journet M., I97o. Influence de la teneur en eau et de la déshydratation de l'herbe sur la valeur alimentaire pour les vaches laitières. Ann. Zootech., 19, 255-268. 